\title{
STRONG SIZE PROPERTIES
}

\author{
Sergio Macías and CÉsar Piceno \\ Universidad Nacional Autónoma de México, Mexico
}

\begin{abstract}
We prove that countable aposyndesis, finite-aposyndesis, continuum chainability, acyclicity (for $n \geq 3$ ), and acyclicity for locally connected continua are strong size properties. As a consequence of our results we obtain that arcwise connectedness is a strong size property which is originally proved by Hosokawa.
\end{abstract}

\section{INTRODUCTION}

Hosokawa defines strong size maps on the $n$-fold hyperspace of a continuum in [5] as a generalization of Whitney maps for the hyperspace of subcontinua of a continuum and proves the existence of such maps ([5, Theorem 2.2]). He also proves that local connectedness ([5, Theorem 3.1]), arcwise connectedness ([5, Theorem 3.3]) and aposyndesis ([5, Theorem 3.4]) are strong size properties. It is natural to ask what other topological properties are strong size properties. We prove that countable aposyndesis (Theorem 4.1), finite-aposyndesis (Corollary 4.2), continuum chainability (Corollary 4.4), acyclicity for $n \geq 3$ (Corollary 4.17), and acyclicity for locally connected continua (Corollary 4.18) are strong size properties. We note that Corollary 4.2 answers one of the questions asked by Hosokawa ([5, Question, p. 964]). As a consequence of Theorem 4.3 we obtain that arcwise connectedness is a strong size property (Corollary 4.5) which is originally proved by Hosokawa. We end the paper with a theorem about extending strong size map defined on a closed subset of the $n$-fold hyperspace of a continuum to the complete $n$-fold hyperspace (Theorem 5.4).

2010 Mathematics Subject Classification. 54B20.

Key words and phrases. Absolute retract, acyclic continuum, continuum, continuum chainable continuum, countable aposyndesis, deformation retract, finite aposyndesis, $n$-fold hyperspace, retract, retraction, strong size level, strong size map, strong size properties. 
Strong size properties are the natural generalization of Whitney properties which have been study extensively in [15, Chapter XIV] and [8, Chapter VIII].

\section{Definitions And Notation}

Given a subset $A$ of a metric space $Z$ with metric $d$, the closure, the boundary and the interior of $A$ are denoted by $C l_{Z}(A), B d_{Z}(A)$ and $\operatorname{Int}_{Z}(A)$, respectively. Also, $\mathcal{V}_{r}(A)$ denotes the open ball of radius $r$ about $A$.

Let $Z$ be a metric space. By a deformation we mean a map $H: Z \times[0,1] \rightarrow$ $Z$ such that $H((z, 0))=z$ for each $z \in Z$. Let $A=\{H((z, 1)) \mid z \in Z\}$. If the map $r: Z \rightarrow A$ given by $r(z)=H((z, 1))$ is a retraction from $Z$ onto $A$, then $H$ is a deformation retraction from $Z$ onto $A$. If $H$ is a deformation retraction from $Z$ onto $A$ and for each $a \in A$ and each $t \in[0,1], H((a, t))=a$, then $H$ is a strong deformation retraction from $Z$ onto $A$. The set $A$ is called a deformation retract of $Z$ (strong deformation retract of $Z$, respectively). A metric space $Z$ is an absolute retract provided that for each embedding $e: Z \rightarrow X$ of $Z$ into a metric space $X$ such that $e(Z)$ is closed in $X, e(Z)$ is a retract of $X$.

A map is a continuous function. The symbol $\rightarrow$ denotes a surjective map.

A continuum is a nonempty compact connected metric space. A continuum $X$ is aposyndetic provided that for each pair of points $x_{1}$ and $x_{2}$ of $X$, there exists a subcontinuum $W$ of $X$ such that $x_{1} \in \operatorname{Int}_{X}(W) \subset W \subset X \backslash\left\{x_{2}\right\}$. The continuum $X$ is finitely aposyndetic (countable aposyndetic) if for each finite (countable closed) subset $F$ of $X$ and each point $x \in X \backslash F$, there exists a subcontinuum $W$ of $X$ such that $x \in \operatorname{Int}_{X}(W) \subset W \subset X \backslash F$.

Let $p$ and $q$ be two points of the continuum $X$. A finite collection $\left\{L_{1}, \ldots, L_{m}\right\}$ of sets is called a chain from $p$ to $q$ provided that $p \in L_{1}$, $q \in L_{m}$ and $L_{j} \cap L_{k} \neq \emptyset$ if and only if $|j-k| \leq 1$. A chain is called a continuum chain if each of its elements is a continuum. A continuum chain is an $\varepsilon$-continuum chain if the diameter of each of its elements is less than $\varepsilon$. The continuum $X$ is said to be continuum chainable provided that for each pair of points $p$ and $q$ of $X$ and each $\varepsilon>0$, there exists an $\varepsilon$-continuum chain from $p$ to $q$ in $X$.

REMARK 2.1. Observe that any arcwise connected continuum is a continuum chainable continuum.

If $X$ is a continuum and $n$ is a positive integer, then $\check{H}^{n}(X)$ denotes the reduced $n$th Cech cohomology group of $X$ with integer coefficients. A continuum $X$ is said to be acyclic if $\check{H}^{1}(X)$ is trivial.

Given a continuum $X$, we consider the following hyperspaces of $X$ :

$$
2^{X}=\{A \subset X \mid A \text { is nonempty and closed }\}
$$

and

$$
\mathcal{C}_{n}(X)=\left\{A \in 2^{X} \mid A \text { has at most } n \text { components }\right\}
$$


where $n$ is a positive integer. $\mathcal{C}_{n}(X)$ is called the $n$-fold hyperspace of $X$. These spaces are topologized with the Hausdorff metric defined as follows:

$$
\mathcal{H}(A, B)=\inf \left\{\varepsilon>0 \mid A \subset \mathcal{V}_{\varepsilon}(B) \text { and } B \subset \mathcal{V}_{\varepsilon}(A)\right\},
$$

$\mathcal{H}$ always denotes the Hausdorff metric on $2^{X}$. When $n=1$, we write $\mathcal{C}(X)$ instead of $\mathcal{C}_{1}(X)$. Given an element $B$ of $\mathcal{C}_{n}(X)$, the mesh of $B$, denoted by $\operatorname{mesh}(B)$, is

$$
\operatorname{mesh}(B)=\max \{\operatorname{diam}(K) \mid K \text { is a component of } B\} .
$$

The symbol $\mathcal{F}_{n}(X)$ denotes the $n$-fold symmetric product of $X$; that is:

$$
\mathcal{F}_{n}(X)=\left\{A \in \mathcal{C}_{n}(X) \mid A \text { has at most } n \text { points }\right\} .
$$

Note that, by definition, $\mathcal{F}_{n}(X) \subset \mathcal{C}_{n}(X)$. It is known that if $X$ is a continuum, then $2^{X}$ and $\mathcal{C}_{n}(X)$ are arcwise connected continua (for $2^{X}$ and $\mathcal{C}(X)$ see $[15,(1.13)]$; for $\mathcal{C}_{n}(X)$ and $n \geq 2$, see [11, 1.8.12]). Also, $\mathcal{F}_{n}(X)$ is a continuum for all positive integers $n$ ([1, p. 877]).

Let $X$ be a continuum and let $n$ be a positive integer. An order arc in $\mathcal{C}_{n}(X)$ is an $\operatorname{arc} \alpha:[0,1] \rightarrow \mathcal{C}_{n}(X)$ such that if $0 \leq s<t \leq 1$, then $\alpha(s) \subset \alpha(t)$ and $\alpha(s) \neq \alpha(t)$.

Let $B$ and $A$ be two elements of $\mathcal{C}_{n}(X)$. We say that the pair $(B, A)$ satisfies property $(O A)$ provided that $B \subset A$ and each component of $A$ intersects $B$. Let us note that this condition guaratees the existence of an order arc, in $\mathcal{C}_{n}(X)$, from $B$ to $A$ when $B \subset A$ and $B \neq A[15,(1.8)]$.

Let $X$ be a continuum and let $n$ be a positive integer. If $B \in \mathcal{C}_{n}(X)$, define:

$$
\mathcal{C}_{n}(B, X)=\left\{A \in \mathcal{C}_{n}(X) \mid B \subset A\right\}
$$

$$
\mathcal{O A}_{n}(B, X)=\left\{A \in \mathcal{C}_{n}(X) \mid(B, A) \text { satisfies property }(O A)\right\}
$$

If $A \in \mathcal{O} \mathcal{A}_{n}(B, X)$, then

$$
\mathcal{O A}_{n}(B, A)=\left\{D \in \mathcal{O} \mathcal{A}_{n}(B, X) \mid D \subset A\right\} .
$$

A map $\mu: \mathcal{C}_{n}(X) \rightarrow[0, \infty)$ is said to be a strong size map provided that:

(1) $\mu(A)=0$ for every $A \in \mathcal{F}_{n}(X)$;

(2) if $A \subset B, A \neq B$ and $B \notin \mathcal{F}_{n}(X)$, then $\mu(A)<\mu(B)$.

Since $X$ is nondegenerate, we may assume that $\mu(X)=1$. By [5, Theorem 2.2 ], strong size maps exist for each continuum $X$ and each positive integer $n$. Note that for $n=1$ a strong size map is just a Whitney map.

Each set of the form $\mu^{-1}(t)$ for any strong size map $\mu$ for $\mathcal{C}_{n}(X)$ and any $t \in[0,1]$ is called a strong size level of $\mathcal{C}_{n}(X)$.

A topological property $P$ is called a strong size property if whenever $X$ has property $P$, so does every strong size level of $\mathcal{C}_{n}(X)$ for each positive integer $n$. 


\section{Preliminary Results}

LEMMA 3.1. Let $X$ be a continuum, let $n$ be a positive integer and let $\mu: \mathcal{C}_{n}(X) \rightarrow[0,1]$ be a strong size map. If $t>0$ and $\mathcal{S}=\mu^{-1}(t)$ is a strong size level for $\mathcal{C}_{n}(X), \mathcal{A}$ is a closed subset of $\mathcal{S}$ and $B \in \mathcal{S} \backslash \mathcal{A}$, then there exists $a \varepsilon>0$ such that $A \not \subset \mathcal{V}_{\varepsilon}(B)$ for any $A \in \mathcal{A}$.

Proof. Suppose the result is not true. Then for each positive integer $m$, there exists $A_{m} \in \mathcal{A}$ such that $A_{m} \subset \mathcal{V}_{\frac{1}{m}}(B)$. Since $\mathcal{A}$ is closed, without loss of generality, we assume that the sequence $\left\{A_{m}\right\}_{m=1}^{\infty}$ converges to an element $A$ of $\mathcal{A}$. Note that $A \subset B$. Since $\mathcal{S}$ a strong size level, we have that $A=B$. Hence, $B \in \mathcal{A}$, a contradiction to the election of $B$. Therefore, the lemma is true.

The proof of the following lemma is similar to the one done for a similar result for Whitney levels $([15,(14.8 .1)])$; we include the details for completeness.

Lemma 3.2. Let $X$ be a continuum, let $n$ be a positive integer, let $\mu: \mathcal{C}_{n}(X) \rightarrow[0,1]$ be a strong size map, and let $\mathcal{S}=\mu^{-1}(t)$ be a strong size level for $\mathcal{C}_{n}(X)$. Let $A, B \in \mathcal{S}$, let $A_{1}, \ldots, A_{\ell}$ be the components of $A$ and let $B_{1}, \ldots, B_{m}$ be the components of $B$. If $P \in \mathcal{F}_{n}(X)$ is such that $P \subset A \cap B$, $P \cap A_{j} \neq \emptyset$ for each $j \in\{1, \ldots, \ell\}$ and $P \cap B_{k} \neq \emptyset$ for all $k \in\{1, \ldots, m\}$, then there exists an arc in $\mathcal{S}$ joining $A$ and $B$.

Proof. Let $\alpha, \beta:[0,1] \rightarrow \mathcal{C}_{n}(X)$ be two order arcs such that $\alpha(0)=P$, $\alpha(1)=A, \beta(0)=P$ and $\beta(1)=B([2$, Proposition 2.6]). Given $s \in[0,1]$, define $f_{s}:[0,1] \rightarrow \mathcal{C}_{n}(X)$ by $f_{s}(r)=\alpha(s) \cup \beta(r)$. Then $f_{s}$ is well defined and continuous. Since $\mu\left(f_{s}(0)\right)=\mu(\alpha(s) \cup \beta(0))=\mu(\alpha(s)) \leq t$ and $\mu\left(f_{s}(1)\right)=$ $\mu(\alpha(s) \cup \beta(1))=\mu(\alpha(s) \cup B) \geq t$, there exists $r_{s} \in[0,1]$ such that $\mu\left(f_{s}\left(r_{s}\right)\right)=$ $t$.

Let $\gamma:[0,1] \rightarrow \mathcal{S}$ be given by $\gamma(s)=\alpha(s) \cup \beta\left(r_{s}\right)$. We show $\gamma$ is well defined. To this end, let $s \in[0,1]$ and suppose there exists $r \in[0,1]$ such that $\alpha(s) \cup \beta(r) \in \mathcal{S}$. Since $\beta$ is an order arc, we have that either $\beta(r) \subset \beta\left(r_{s}\right)$ or $\beta\left(r_{s}\right) \subset \beta(r)$. Without loss of generality we assume that $\beta(r) \subset \beta\left(r_{s}\right)$. Then $\alpha(s) \cup \beta(r) \subset \alpha(s) \cup \beta\left(r_{s}\right)$. Since $\mu$ is a strong size map, we obtain that $\alpha(s) \cup \beta(r)=\alpha(s) \cup \beta\left(r_{s}\right)$. Thus, $\gamma$ is well defined.

To see that $\gamma$ is continuous, let $\left\{s_{m}\right\}_{m=1}^{\infty}$ be sequence of elements of $[0,1]$ converging to an element $s$ of $[0,1]$. Then the corresponding sequence $\left\{r_{s_{m}}\right\}_{m=1}^{\infty}$ has a convergent subsequence $\left\{r_{s_{m_{k}}}\right\}_{k=1}^{\infty}$. Let $r$ be the limit of the sequence $\left\{r_{s_{m_{k}}}\right\}_{k=1}^{\infty}$. Since $\alpha$ and $\beta$ are continuous, we have that $\lim _{k \rightarrow \infty} \gamma\left(s_{m_{k}}\right)=\lim _{k \rightarrow \infty}\left(\alpha\left(s_{m_{k}}\right) \cup \beta\left(r_{s_{m_{k}}}\right)\right)=\alpha(s) \cup \beta(r)$. By definition of $\gamma$, $\gamma(s)=\alpha(s) \cup \beta\left(r_{s}\right)$. Since both $\alpha(s) \cup \beta(r)$ and $\alpha(s) \cup \beta\left(r_{s}\right)$ belong to $\mathcal{S}$ and either $\alpha(s) \cup \beta(r) \subset \alpha(s) \cup \beta\left(r_{s}\right)$ or $\alpha(s) \cup \beta\left(r_{s}\right) \subset \alpha(s) \cup \beta(r)$, we have that $\alpha(s) \cup \beta(r)=\alpha(s) \cup \beta\left(r_{s}\right)$. Therefore, $\gamma$ is continuous. 
It seems that the following lemma is well known but we cannot find a reference for it.

LEMma 3.3. If $X$ is a continuum, then $\check{H}^{0}(X)$ is trivial.

PRoOF. The result follows form three the facts: (1) each continuum is an inverse limit of connected polyhedra ([13, Theorem 2]), (2) the 0th reduced cohomology group of a connected polyhedron is trivial ([14, 42.2]), and (3) the continuity theorem for Cech cohomology ([18, Theorem 7-7]).

\section{Strong Size Properties}

A topological property $P$ is called a strong size property if whenever $X$ has property $P$, so does every strong size level of $\mathcal{C}_{n}(X)$ for each positive integer $n$.

H. Hosokawa proves in [5, Theorem 3.4] that aposyndesis is a strong size property, we extend this result to countable aposyndesis.

THEOREM 4.1. Countable aposyndesis is a strong size property.

Proof. Let $X$ be a countable aposyndetic continuum and let $\mu: \mathcal{C}_{n}(X) \rightarrow[0,1]$ be a strong size map, let $t \in[0,1]$ and let $\mathcal{S}=\mu^{-1}(t)$ be a strong size level for $\mathcal{C}_{n}(X)$. It is known that if $n \geq 2$, then $\mathcal{F}_{n}(X)$ is countable aposyndetic [10, Theorem 8]. Hence, the case $t=0$ follows. Suppose $t>0$ and let $\mathcal{A}$ be a countable closed subset of $\mathcal{S}$. Let $B \in \mathcal{S} \backslash \mathcal{A}$. By Lemma 3.1, there exists $\varepsilon>0$ such that $A \not \subset \mathcal{V}_{\varepsilon}(B)$ for any $A \in \mathcal{A}$. Let $U=X \backslash C l_{X}\left(\mathcal{V}_{\frac{\varepsilon}{2}}(B)\right)$. By $[7$, Theorem 2.1], there exists a map $s: \mathcal{A} \rightarrow X$ such that $s(A) \in A \cap U$ for each $A \in \mathcal{A}$. Hence, $s(\mathcal{A})$ is a countable closed subset of $X$ such that $s(\mathcal{A}) \cap B=\emptyset$. Then for each $b \in B$, there exists a subcontinuum $K_{b}$ of $X$ such that $b \in \operatorname{Int}_{X}\left(K_{b}\right) \subset K_{b} \subset X \backslash s(\mathcal{A})$. Thus, $\left\{\operatorname{Int}_{X}\left(K_{b}\right) \mid b \in B\right\}$ is an open cover of $B$. Since $B$ is compact, there exist $b_{1}, \ldots, b_{\ell} \in B$ such that $B \subset \cup_{j=1}^{\ell} \operatorname{Int}_{X}\left(K_{b_{j}}\right) \subset \cup_{j=1}^{\ell} K_{b_{j}}$. Without loss of generality, we assume that the family $\left\{K_{b_{1}}, \ldots, K_{b_{\ell}}\right\}$ consists of pairwise disjoint continua. Hence, by [5, Theorem 2.14], $\left\langle K_{b_{1}}, \ldots, K_{b_{\ell}}\right\rangle \cap \mathcal{S}$ is a subcontinuum of $\mathcal{S}$. Note that $B \in \operatorname{Int}_{\mathcal{C}_{n}(X)}\left(\left\langle K_{b_{1}}, \ldots, K_{b_{\ell}}\right\rangle\right) \cap \mathcal{S}$. Since for each $j \in\{1, \ldots, \ell\}, s(\mathcal{A}) \cap K_{b_{j}}=\emptyset$, we obtain that $A \not \subset \cup_{j=1}^{\ell} K_{b_{j}}$ for any $A \in \mathcal{A}$. Hence, $\left(\left\langle K_{b_{1}}, \ldots, K_{b_{\ell}}\right\rangle \cap \mathcal{S}\right) \cap \mathcal{A}=\emptyset$. Therefore, $\mathcal{S}$ is countable aposyndetic.

The following corollary answers one of the questions of Hosokawa [5, Question, p. 964].

COROLlary 4.2. Finite aposyndesis is a strong size property.

TheOREM 4.3. Let $X$ be a continuum chainable continuum, let $n$ be a positive integer and let $\mu: \mathcal{C}_{n}(X) \rightarrow[0,1]$ be a strong size map. If $t \in(0,1)$ and $\mathcal{S}=\mu^{-1}(t)$, then $\mathcal{S}$ is arcwise connected. 
Proof. Let $A$ and $B$ be two elements of $\mathcal{S}$. Since $\mathcal{C}_{n}(X)$ is compact and $\mu$ is continuous, there exists $\varepsilon>0$ such that if $D \in \mathcal{C}_{n}(X)$ and $\operatorname{mesh}(D)<\varepsilon$, then $\mu(D)<t$. Since $t>0$ and $A, B \in \mathcal{S}$, at least one of the components of $A$ and $B$ is nondegenerate. Hence, $A$ and $B$ have uncountably many points. Let $\left\{a_{1}, \ldots, a_{n}\right\}$ be a subset of $A$ such that it intersects each component of $A$. Similarly, let $\left\{b_{1}, \ldots, b_{n}\right\}$ be a subset of $B$ such that it intersects each component of $B$.

Since $X$ is a continuum chainable continuum, for each $i \in\{1, \ldots, n\}$, there exist subcontinua $D_{1}^{i}, \ldots, D_{k_{i}}^{i}$ of $X$ such that $a_{i} \in D_{1}^{i}, b_{i} \in D_{k_{i}}^{i}, D_{j}^{i} \cap D_{\ell}^{i} \neq \emptyset$ if and only if $|j-\ell| \leq 1$, and $\operatorname{diam}\left(D_{j}^{i}\right)<\frac{\varepsilon}{n}$ for $j \in\left\{1, \ldots, k_{i}\right\}$. Let $k=$ $\max \left\{k_{1}, \ldots, k_{n}\right\}$. For each $j \in\{1, \ldots, k\}$, let $D_{j}=\cup_{i=1}^{n} D_{j}^{i}$, where $D_{j}^{i}=D_{k_{i}}^{i}$ if $j \geq k_{i}$. Note that for every $j \in\{1, \ldots, k\}, D_{j} \in \mathcal{C}_{n}(X)$ and $\operatorname{mesh}\left(D_{j}\right)<\varepsilon$. Hence, $\mu\left(D_{j}\right)<t$ for all $j \in\{1, \ldots, k\}$. For each $j \in\{1, \ldots, k-1\}$, let $p_{j}^{i} \in D_{j}^{i} \cap D_{j+1}^{i}$ and let $P_{j}=\left\{p_{j}^{1}, \ldots, p_{j}^{n}\right\}$.

For each $j \in\{1, \ldots, k\}$, let $\alpha_{j}$ be an order arc from $D_{j}$ to $X$ and let $D_{j}^{\prime} \in \mathcal{S}$ be such that $\left\{D_{j}^{\prime}\right\}=\alpha_{j} \cap \mathcal{S}$. Note that $A, D_{1}^{\prime}$ and $\left\{a_{1}, \ldots, a_{n}\right\}$ satisfy the hypothesis of Lemma 3.2. Then there exists an $\operatorname{arc} \beta_{1}$ in $\mathcal{S}$ from $A$ to $D_{1}^{\prime}$. Also note that if $j \in\{1, \ldots, k-1\}$, then $D_{j}^{\prime}, D_{j+1}^{\prime}$ and $P_{j}$ satisfy the hypothesis of Lemma 3.2. Thus, there exists an arc $\beta_{j+1}$ in $\mathcal{S}$ from $D_{j}^{\prime}$ to $D_{j+1}^{\prime}$. Similarly, by Lemma 3.2 , there exists an arc $\beta_{k+1}$ in $\mathcal{S}$ from $D_{k}^{\prime}$ to $B$. Hence, $\cup_{j=1}^{k+1} \beta_{j}$ contains an arc from $A$ to $B$. Therefore, $\mathcal{S}$ is arcwise connected.

We have the following:

COROLlary 4.4. Being a continuum chainable continuum is a strong size property.

Proof. Let $X$ be a continuum chainable continuum and let $\mu: \mathcal{C}_{n}(X) \rightarrow[0,1]$ be a strong size map, let $t \in[0,1]$ and let $\mathcal{S}=\mu^{-1}(t)$ be a strong size level for $\mathcal{C}_{n}(X)$. It is known that $X$ is continuum chainable if and only if $\mathcal{F}_{n}(X)$ is continuum chainable for each positive integer $n$ ([2, Theorem $2.9])$. Hence, the case $t=0$ follows. For $t>0$, the result follows from Remark 2.1 and Theorem 4.3.

As a consequence of Remark 2.1, Theorem 4.3 and [2, Proposition 2.7], we obtain the following result of Hosokawa ([5, Theorem 3.3]):

COROLlary 4.5. Being an arcwise connected continuum is a strong size property.

Our next goal is to prove that for an integer $n \geq 3$, the strong size levels of $\mathcal{C}_{n}(X)$ are acyclic (Corollary 4.17). To this end, we follow [17]. We include all the details for the convenience of the reader.

Let us mention that it is known that acyclicity is not a Whitney property ([16, Example 2]) and it is for 1-dimensional continua ([17, Corollary 7$])$. Since 
we do not ask any additional properties to the continuum $X$, Theorem 4.16 says that, for $n \geq 3$, the levels of strong size maps are much nicer than the ones of Whitney maps. In particular, Corollary 4.17, tells us the acyclicity is a strong size property.

A nonempty collection $\Sigma$ of closed subsets of a continuum $X$ is called a structure if $\Sigma$ is closed with respect to finite unions, finite intersections, and intersections of towers ordered by inclusion. If $\Sigma$ is a structure on $X$, then an element $P$ of $\Sigma$ is called an indecomposable set provided that whenever $P=A \cup B$, for some elements $A$ and $B$ of $\Sigma$, we have that $P=A$ or $P=B$.

Given a continuum $X$, we consider two structures in $\mathcal{C}_{n}(X)$. If $\mathcal{B}$ is a closed subset of $\mathcal{C}_{n}(X)$, let

$$
\mathcal{M}(\mathcal{B})=\cup\left\{\mathcal{O}_{n}(B, X) \mid B \in \mathcal{B}\right\} .
$$

REmark 4.6. Note that if $\mathcal{B}$ and $\mathcal{D}$ are two closed subsets of $\mathcal{C}_{n}(X)$, then $\mathcal{M}(\mathcal{B} \cup \mathcal{D})=\mathcal{M}(\mathcal{B}) \cup \mathcal{M}(\mathcal{D})$ and $\mathcal{M}(\mathcal{B}) \cap \mathcal{M}(\mathcal{D})=\mathcal{M}(\mathcal{M}(\mathcal{B}) \cap \mathcal{M}(\mathcal{D}))$. Also, if $\left\{\mathcal{B}_{\lambda}\right\}_{\lambda \in \Lambda}$ is a tower ordered by inclusion, then $\mathcal{M}\left(\cap_{\lambda \in \Lambda} \mathcal{M}\left(\mathcal{B}_{\lambda}\right)\right)=$ $\cap_{\lambda \in \Lambda} \mathcal{M}\left(\mathcal{B}_{\lambda}\right)$.

Lemma 4.7. Let $X$ be a continuum and let $n$ be a positive integer. If $\mathcal{B}$ is a closed subset of $\mathcal{C}_{n}(X)$, then $\mathcal{M}(\mathcal{B})$ is closed in $\mathcal{C}_{n}(X)$.

Proof. Let $D \in C l_{\mathcal{C}_{n}(X)}(\mathcal{M}(\mathcal{B}))$. Then there exists a sequence $\left\{D_{m}\right\}_{m=1}^{\infty}$ of elements of $\mathcal{M}(\mathcal{B})$ converging to $D$. For each $m$, there exists $B_{m} \in \mathcal{B}$ such that $D_{m} \in \mathcal{O}_{n}\left(B_{m}, X\right)$. Since $\mathcal{B}$ is compact, there exists a subsequence $\left\{B_{m_{k}}\right\}_{k=1}^{\infty}$ of the sequence $\left\{B_{m}\right\}_{m=1}^{\infty}$ that converges to an element $B$ of $\mathcal{B}$. Since $D_{m_{k}} \in \mathcal{O} \mathcal{A}_{n}\left(B_{m_{k}}, X\right)$, we have that $D \in \mathcal{O} \mathcal{A}_{n}(B, X)$. Therefore, $\mathcal{M}(\mathcal{B})$ is closed in $\mathcal{C}_{n}(X)$.

For a continuum $X$ and a positive integer $n$, let

$$
\Sigma_{1}=\left\{\mathcal{M}(\mathcal{B}) \mid \mathcal{B} \text { is a closed subset of } \mathcal{C}_{n}(X)\right\} .
$$

By Remark 4.6 and Lemma $4.7, \Sigma_{1}$ is a structure.

REMARK 4.8. Note that the indecomposable sets of $\Sigma_{1}$ are the sets of the form $\mathcal{M}(\{B\})$ where $B \in \mathcal{C}_{n}(X)$. Since $\mathcal{M}(\{B\})$ is homeomorphic to $\mathcal{O} \mathcal{A}_{n}(B, X)$ and this set is an absolute retract $([12,4.3])$, the indecomposable sets of $\Sigma_{1}$ have all its reduced Čech cohomology groups trivial. Hence, all the reduced Cech cohomology groups of each member of $\Sigma_{1}$ are trivial ([17, Theorem 2]).

The second structure is found in $\mathcal{O} \mathcal{A}_{n}(Z, X)$, where $Z$ is an arbitrary point of $\mathcal{C}_{n}(X)$. If $\mathcal{B}$ is a closed subset of $\mathcal{O} \mathcal{A}_{n}(Z, X)$, let

$$
\mathcal{L}(\mathcal{B})=\cup\left\{\mathcal{O}_{n}(Z, B) \mid B \in \mathcal{B}\right\}
$$

Remark 4.9. Observe that if $\mathcal{B}$ and $\mathcal{D}$ are two closed subsets of $\mathcal{C}_{n}(X)$, then $\mathcal{L}(\mathcal{B} \cup \mathcal{D})=\mathcal{L}(\mathcal{B}) \cup \mathcal{L}(\mathcal{D})$ and $\mathcal{L}(\mathcal{B}) \cap \mathcal{L}(\mathcal{D})=\mathcal{L}(\mathcal{L}(\mathcal{B}) \cap \mathcal{L}(\mathcal{D}))$. Also, if $\left\{\mathcal{B}_{\lambda}\right\}_{\lambda \in \Lambda}$ is a tower ordered by inclusion, then $\mathcal{L}\left(\cap_{\lambda \in \Lambda} \mathcal{L}\left(\mathcal{B}_{\lambda}\right)\right)=\cap_{\lambda \in \Lambda} \mathcal{L}\left(\mathcal{B}_{\lambda}\right)$. 
LEMMA 4.10. Let $X$ be a continuum, let $n$ be a positive integer and let $Z \in \mathcal{C}_{n}(X)$. If $\mathcal{B}$ is a closed subset of $\mathcal{O A}_{n}(Z, X)$, then $\mathcal{L}(\mathcal{B})$ is closed in $\mathcal{C}_{n}(X)$.

Proof. Let $D \in C l_{\mathcal{C}_{n}(X)}(\mathcal{L}(\mathcal{B}))$. Then there exists a sequence $\left\{D_{m}\right\}_{m=1}^{\infty}$ of elements of $\mathcal{L}(\mathcal{B})$ converging to $D$. For each $m$, there exists $B_{m} \in \mathcal{B}$ such that $D_{m} \in \mathcal{O} \mathcal{A}_{n}\left(Z, B_{m}\right)$. Since $\mathcal{O} \mathcal{A}_{n}(Z, X)$ is a continuum $([12,4.3])$, $\mathcal{B}$ is compact. Then there exists a subsequence $\left\{B_{m_{k}}\right\}_{k=1}^{\infty}$ of the sequence $\left\{B_{m}\right\}_{m=1}^{\infty}$ that converges to an element $B$ of $\mathcal{B}$. Since $D_{m_{k}} \in \mathcal{O} \mathcal{A}_{n}\left(Z, B_{m_{k}}\right)$, we obtain that $D \in \mathcal{O} \mathcal{A}_{n}(Z, B)$. Therefore, $\mathcal{L}(\mathcal{B})$ is closed in $\mathcal{C}_{n}(X)$.

Let $X$ be a continuum and let $n$ be a positive integer. For an element $Z$ of $\mathcal{C}_{n}(X)$, let

$$
\Sigma_{2}=\left\{\mathcal{L}(\mathcal{B}) \mid \mathcal{B} \text { is a closed subset of } \mathcal{O} \mathcal{A}_{n}(Z, X)\right\} .
$$

By Remark 4.9 and Lemma $4.10, \Sigma_{2}$ is a structure.

REMARK 4.11. Note that the indecomposable sets of $\Sigma_{2}$ are the sets of the form $\mathcal{L}(\{B\})$ where $B \in \mathcal{O} \mathcal{A}_{n}(Z, X)$. Since $\mathcal{L}(\{B\})$ is homeomorphic to $\mathcal{O}_{n}(Z, B)$ and this set is an absolute retract $([12,4.3])$, all the reduced Cech cohomology groups of the indecomposable sets of $\Sigma_{2}$ are trivial. Thus, all the reduced Cech cohomology groups of each member of $\Sigma_{2}$ are trivial ([17, Theorem 2]).

Let $X$ be a continuum, let $n$ be a positive integer and let $\mu: \mathcal{C}_{n}(X) \rightarrow[0,1]$ be a strong size map. For an element $Z$ of $\mathcal{C}_{n}(X)$ and an element $t \in[\mu(Z), 1]$, let

$$
\mathcal{D}_{n}(Z, t)=\mathcal{M}(\{Z\}) \cap \mu^{-1}(t) .
$$

As a consequence of Lemma $3.2, \mathcal{D}_{n}(Z, t)$ is an arcwise connected continuum.

The proof of the following theorem is similar to the one given in $[17$, Theorem 4].

TheOREM 4.12. Let $X$ be a continuum, let $n$ be a positive integer, let $\mu: \mathcal{C}_{n}(X) \rightarrow[0,1]$ be a strong size map and let $Z \in \mathcal{C}_{n}(X)$. If $t \in[\mu(Z), 1]$, then all the reduced $\breve{C}$ ech cohomology groups of $\mathcal{D}_{n}(Z, t)$ are trivial.

Proof. Consider the pair $\left\{\mathcal{M}\left(\mathcal{D}_{n}(Z, t)\right), \mathcal{L}\left(\mathcal{D}_{n}(Z, t)\right)\right\}$ of subsets of $\mathcal{O} \mathcal{A}_{n}(Z, X)$. For an integer $m \geq 0$, consider the following part of the reduced Mayer-Vietoris sequence:

$\check{H}^{m}\left(\mathcal{M}\left(\mathcal{D}_{n}(Z, t)\right)\right) \oplus \check{H}^{m}\left(\mathcal{L}\left(\mathcal{D}_{n}(Z, t)\right)\right) \rightarrow \check{H}^{m}\left(\mathcal{D}_{n}(Z, t)\right) \rightarrow \check{H}^{m+1}\left(\mathcal{O} \mathcal{A}_{n}(Z, X)\right)$

for this pair. By Remarks 4.8 and 4.11, we have that $\check{H}^{m}\left(\mathcal{M}\left(\mathcal{D}_{n}(Z, t)\right)\right)$ and $\check{H}^{m}\left(\mathcal{L}\left(\mathcal{D}_{n}(Z, t)\right)\right)$ are trivial. Since $\mathcal{O} \mathcal{A}_{n}(Z, X)$ is an absolute retract $([12,4.3]), H^{m+1}\left(\mathcal{O} \mathcal{A}_{n}(Z, X)\right)$ is trivial too. Hence, $\check{H}^{m}\left(\mathcal{D}_{n}(Z, t)\right)$ is trivial. Therefore, all the reduced Cech cohomology groups of $\mathcal{D}_{n}(Z, t)$ are trivial. 
Let $X$ be a continuum, let $n$ be a positive integer and let $\mu: \mathcal{C}_{n}(X) \rightarrow[0,1]$ be a strong size map. Let $s, t \in[0,1]$ be such that $s \leq t$. Define ${ }_{n} \gamma_{s}^{t}: \mu^{-1}(s) \rightarrow$ $\mu^{-1}(t)$ by ${ }_{n} \gamma_{s}^{t}(Z)=\mathcal{D}_{n}(Z, t)$. The next lemma shows that ${ }_{n} \gamma_{s}^{t}$ is upper semicontinuous.

LEMma 4.13. Let $X$ be a continuum, let $n$ be a positive integer and let $\mu: \mathcal{C}_{n}(X) \rightarrow[0,1]$ be a strong size map. If $s, t \in[0,1]$ are such that $s \leq t$, then $n \gamma_{s}^{t}$ is upper semicontinuous.

Proof. Let $\left\{Z_{m}\right\}_{m=1}^{\infty}$ be a sequence of elements of $\mu^{-1}(s)$ that converges to an element $Z$ of $\mu^{-1}(s)$. Let $Y \in \lim \sup _{n} \gamma_{s}^{t}\left(Z_{m}\right)$. Then there exists a subsequence $\left\{m_{k}\right\}_{k=1}^{\infty}$ of the natural sequence such that for each positive integer $k$, there exists $Y_{m_{k}} \in{ }_{n} \gamma_{s}^{t}\left(Z_{m_{k}}\right)$ such that the sequence $\left\{Y_{m_{k}}\right\}_{k=1}^{\infty}$ converges to $Y$. Since for all $k Z_{m_{k}} \subset Y_{m_{k}}$ and $\left\{Z_{m_{k}}\right\}_{k=1}^{\infty}$ converges to $Z$, we have that $Z \subset Y$. It is easy to see that $Y \in \mathcal{O}_{n}(Z, X)$. Hence, $Y \in_{n} \gamma_{s}^{t}(Z)$. Therefore, ${ }_{n} \gamma_{s}^{t}$ is upper semicontinuous.

THEOREM 4.14. Let $X$ be a continuum, let $n$ be a positive integer and let $\mu: \mathcal{C}_{n}(X) \rightarrow[0,1]$ be a strong size map. If $s, t \in[0,1]$ are such that $s \leq t$, then ${ }_{n} \gamma_{s}^{t}$ induces a monomorphism $\left({ }_{n} \gamma_{s}^{t}\right)^{*}: \check{H}^{1}\left(\mu^{-1}(t)\right) \rightarrow \check{H}^{1}\left(\mu^{-1}(s)\right)$.

Proof. By Theorem 4.12, all the reduced Cech cohomology groups of ${ }_{n} \gamma_{s}^{t}(Z)$ are trivial. Suppose $t \neq 1$, the result is clear for $t=1$. Let $B \in$ $\mu^{-1}(t)$ and suppose that $B_{1}, \ldots, B_{m}$ are the components of $B$. Note that $\left({ }_{n} \gamma_{s}^{t}\right)^{-1}(B)=\left\langle B_{1}, \ldots, B_{m}\right\rangle_{n} \cap \mu^{-1}(s)$ and this set is a proper continuum of $\mu^{-1}(s)$ by [5, Theorem 2.14]. The result now follows from Lemmas 4.13, 3.3 and $[17$, Theorem 3].

Corollary 4.15. Let $X$ be a continuum, let $n$ be a positive integer and let $\mu: \mathcal{C}_{n}(X) \rightarrow[0,1]$ be a strong size map. If $t \in[0,1]$, then ${ }_{n} \gamma_{0}^{t}$ induces a monomorphism $\left({ }_{n} \gamma_{0}^{t}\right)^{*}: \check{H}^{1}\left(\mu^{-1}(t)\right) \rightarrow \check{H}^{1}\left(\mu^{-1}(0)\right)$.

Theorem 4.16. Let $X$ be a continuum, let $n \geq 3$ be an integer and let $\mu: \mathcal{C}_{n}(X) \rightarrow[0,1]$ be a strong size map. If $\mathcal{S}=\mu^{-1}(t)$ is a strong size level, then $\mathcal{S}$ is acyclic.

Proof. By [9, Theorem 8], each map from $\mathcal{F}_{n}(X)$ into the unit circle in the plane is homotopic to a constant map. This implies, by [3, 8.1], that $\check{H}^{1}\left(\mathcal{F}_{n}(X)\right)$ is trivial; i.e., $\mathcal{F}_{n}(X)$ is acyclic. The theorem now follows from the fact that $\mu^{-1}(0)=\mathcal{F}_{n}(X)$ and Corollary 4.15.

COROLlaRY 4.17. The property of being acyclic is a strong size property for each integer $n \geq 3$.

COROLlary 4.18. The property of being acyclic is a strong size property for locally connected continua. 
Proof. Let $X$ be a locally connected continuum. For $n \geq 3$, the corollary follows from Corollary 4.17. Suppose $n=2$. By [4, Satz 1], [20, (7.4)] and $[3,8.1]$, we have that $\mathcal{F}_{2}(X)$ is acyclic. Hence, since $\mu^{-1}(0)=\mathcal{F}_{2}(X)$, by Corollary $4.15, \mu^{-1}(t)$ is acyclic for all $t \in(0,1]$. If $n=1$, the corollary follows from $[6, p .253],[20,(7.4)]$ and $[3,8.1]$. Therefore, the property of being acyclic is a strong size property for locally connected continua.

\section{Extending Strong Size Maps}

Let $X$ be a continuum and let $n$ be a positive integer. We show that if $\mathfrak{C}$ is a nonempty closed subset of $\mathcal{C}_{n}(X)$ and $\mu: \mathfrak{C} \rightarrow[0,1]$ is a strong size map, then $\mu$ can be extended to a strong size map defined on $\mathcal{C}_{n}(X)$. To this end, we follow [19].

If $P$ is a partially ordered space and $x \in P$, we write $L(x)=\{p \in P \mid p \leq$ $x\}$ and $M(x)=\{p \in P \mid x \leq p\}$, and if $A \subset P$ then $L(A)=\cup\{L(a) \mid a \in A\}$ and $M(A)=\cup\{M(a) \mid a \in A\}$. An element $m$ of a partially ordered space $P$ is minimal (maximal) if, whenever $x \in P$ and $x \leq m(m \leq x)$, it follows that $m=x$. The set of minimal elements of $P$ is denoted by $\min (P)$ and the set of maximal elements of $P$ is denoted by $\max (P)$.

Recall that given a nondegenerate continuum $X, H$. Hosokawa [5] defined the following order on $\mathcal{C}_{n}(X)$ : For $A, B \in \mathcal{C}_{n}(X)$, define $A<B$ if $A \subset$ $B, A \neq B$ and $B \notin \mathcal{F}_{n}(X)$. We denote $A \leq B$ if $A<B$ or $A=B$. Then $\mathcal{C}_{n}(X)$ is a partially ordered space with respect to this order. Clearly $\min \left(\mathcal{C}_{n}(X)\right)=\mathcal{F}_{n}(X) ; \max \left(\mathcal{C}_{n}(X)\right)=\{X\}$ and these sets are closed and since $X$ is a nondegenerate continuum, they are disjoint.

The following three theorems are Theorems 2.2, 2.3 and Lemma 3.2 of [19]:

THEOREM 5.1. If $K$ is a compact subset of a partially ordered space, then $L(K)$ and $M(K)$ are closed sets.

THEOREM 5.2. If $x$ and $y$ are elements of a compact partially ordered space and if $M(x) \cap L(y)=\emptyset$, then there are disjoint open sets $U$ and $V$ such that $x \in U=M(U)$ and $y \in V=L(V)$.

THEOREM 5.3. Suppose $P$ is a compact partially ordered space such that $\min (P)$ and $\max (P)$ are disjoint closed sets, $Q$ is a closed subset containing $(\min (P)) \cup(\max (P))$, and suppose $A$ and $B$ are disjoint nonempty closed subsets such that $A=M(A)$ and $B=L(B)$. If $f: Q \rightarrow[0,1]$ is a continuous order-preserving function such that $f(\min (P))=\{0\}$ and $f(\max (P))=\{1\}$, then $f$ admits a continuous order-preserving extension $\hat{f}: P \rightarrow[0,1]$ such that $\hat{f}(a) \geq \inf f(A \cap Q)$ for each $a \in A$ and $\hat{f}(b) \leq \sup f(B \cap Q)$ for each $b \in B$.

The proof of the following theorem is similar to the one given for $[19$, Theorem 3.1]; we include the appropriate changes for the convenience of the reader. 
THEOREM 5.4. Let $X$ be a continuum and let $n$ be a positive integer. If $\mathfrak{C}$ is a nonempty closed subset of $\mathcal{C}_{n}(X)$ and $\mu: \mathfrak{C} \rightarrow[0,1]$ is a strong size map, then $\mu$ can be extended to a strong size map $\mu_{n}$ defined on $\mathcal{C}_{n}(X)$.

Proof. Let $\mathfrak{C}$ be a nonempty closed subset of $\mathcal{C}_{n}(X)$ and let $\mu: \mathfrak{C} \rightarrow[0,1]$ be a strong size map. Without loss of generality we assume that $\mathcal{F}_{n}(X) \cup$ $\{X\} \subset \mathfrak{C}$ (if this is not true, let $\mathfrak{K}=\mathfrak{C} \cup \mathcal{F}_{n}(X) \cup\{X\}$ and note that $\mu$ can be extended to a strong size map $\mu^{\prime}$ on $\mathfrak{K}$ by defining $\mu^{\prime}(X)=1$ and $\mu^{\prime}(A)=0$ for each $\left.A \in \mathcal{F}_{n}(X)\right)$.

Let $\mathfrak{U}$ be a countable base for $\mathcal{C}_{n}(X)$ and let

$$
\mathfrak{B}=\{(\mathcal{U}, \mathcal{V}) \mid M(C l(\mathcal{U})) \cap L(C l(\mathcal{V}))=\emptyset \text { and } \mathcal{U}, \mathcal{V} \in \mathfrak{U}\}
$$

Then $\mathfrak{B}$ is countable and we may enumerate its elements $\mathfrak{B}=\left\{\left(\mathcal{U}_{k}, \mathcal{V}_{k}\right) \mid\right.$ $k$ is a positive integer $\}$. By Theorem 5.1 the sets $M(C l(\mathcal{U}))$ and $L(C l(\mathcal{V}))$ are closed. Hence, by Theorem 5.3, for each positive integer $k$, there exists a continuous order-preserving function $\omega_{k}: \mathcal{C}_{n}(X) \rightarrow[0,1]$ such that $\left.\omega_{k}\right|_{\mathfrak{C}}=\mu$ and:

$$
\begin{aligned}
& \omega_{k}(A) \geq \inf \mu\left(M\left(C l\left(\mathcal{U}_{k}\right)\right) \cap \mathfrak{C}\right) \text { if } A \in M\left(C l\left(\mathcal{U}_{k}\right)\right), \\
& \omega_{k}(B) \leq \max \mu\left(L\left(C l\left(\mathcal{V}_{k}\right)\right) \cap \mathfrak{C}\right) \text { if } B \in L\left(C l\left(\mathcal{V}_{k}\right)\right) .
\end{aligned}
$$

Define $\mu_{n}: \mathcal{C}_{n}(X) \rightarrow[0,1]$ by $\mu_{n}(A)=\sum_{k=1}^{\infty} \frac{1}{2^{k}} \omega_{k}(A)$ for all $A \in \mathcal{C}_{n}(X)$. Observe that $\mu_{n}$ is a continuous extension of $\mu$. Since each $\omega_{k}$ is orderpreserving, $\mu_{n}$ is also order preserving.

We need to show that if $A, B \in \mathcal{C}_{n}(X)$ and $A<B$ (in Hosokawa's sense) then $\mu_{n}(A)<\mu_{n}(B)$. It suffices to prove that there exists a positive integer $k$ such that $\omega_{k}(A)<\omega_{k}(B)$.

Let $t_{A}=\sup \mu(L(A) \cap \mathfrak{C})$ and let $t_{B}=\inf \mu(M(B) \cap \mathfrak{C})$. Since $\mu$ is a strong size map, $t_{A}<t_{B}$. Let $\varepsilon>0$ be such that $\varepsilon<\frac{1}{2}\left(t_{B}-t_{A}\right)$. By Theorem 5.2, there exist two disjoint open subsets $\mathcal{U}$ and $\mathcal{V}$ of $\mathcal{C}_{n}(X)$ such that $A \in \mathcal{V}=L(\mathcal{V})$ and $B \in \mathcal{U}=M(\mathcal{U})$ and, by compactness, we may assume that $\mu(\mathcal{V} \cap \mathfrak{C}) \subset\left[0, t_{A}+\varepsilon\right)$ and $\mu(\mathcal{U} \cap \mathfrak{C}) \subset\left(t_{B}-\varepsilon, 1\right]$. It follows that there is a positive integer $k$ such that $A \in \mathcal{V}_{k} \subset C l\left(\mathcal{V}_{k}\right) \subset \mathcal{V}$ and $B \in \mathcal{U}_{k} \subset C l\left(\mathcal{U}_{k}\right) \subset \mathcal{U}$, from here we obtain:

$$
\omega_{k}(A) \leq t_{A}+\varepsilon<t_{B}-\varepsilon \leq \omega_{k}(B) .
$$

Corollary 5.5. Let $X$ be a continuum. If $\mu: \mathcal{C}(X) \rightarrow[0,1]$ is a Whitney map, $\mu$ can be extended to a strong size map $\mu_{n}$ defined on $\mathcal{C}_{n}(X)$.

ACKnowledgements.

The authors thank the referee for the very careful reading of the paper. 


\section{REFERENCES}

[1] K. Borsuk and S. Ulam, On symmetric products of topological spaces, Bull. Amer. Math. Soc. 37 (1931), 875-882.

[2] J. J. Charatonik and S. Macías, Mappings of some hyperspaces, JP J. Geom. Topol. 4 (2004), 53-80.

[3] C. H. Dowker, Mapping theorems for non-compact spaces, Amer. J. Math. 69 (1947), 200-242.

[4] T. Ganea, Symmetrische Potenzen topologischer Räume, Math. Nachr. 11 (1954), 305-316.

[5] H. Hosokawa, Strong size levels of $\mathcal{C}_{n}(X)$, Houston J. Math. 37 (2011), 955-965.

[6] A. Illanes, Multicoherence of Whitney levels, Topology Appl. 68 (1996), 251-265.

[7] A. Illanes, Countable closed set aposyndesis and hyperspaces, Houston J. Math. 23 (1997), 57-64.

[8] A. Illanes and S. B. Nadler, Jr., Hyperspaces. Fundamentals and recent advances, Marcel Dekker, New York, 1999.

[9] S. Macías, On symmetric products of continua, Topology Appl. 92 (1999), 173-182.

[10] S. Macías, Aposyndetic properties of symmetric products of continua, Topology Proc. 22 (1997), 281-296.

[11] S. Macías, Topics on continua, Chapman \& Hall/CRC, Boca Raton, 2005.

[12] S. Macías, Deformation retracts and Hilbert cubes in $n$-fold hyperspaces, Topology Proc. 40 (2012), 215-226.

[13] S. Mardešić and J. Segal, ع-mappings onto polyhedra, Trans. Amer. Math. Soc. 109 (1963), 146-164.

[14] J. R. Munkres, Elements of algebraic topology, Addison-Wesley, Menlo Park, 1984.

[15] S. B. Nadler, Jr., Hyperspaces of sets, Sociedad Matemática Mexicana, México, 2006.

[16] A. Petrus, Contractibility of Whitney continua in $\mathcal{C}(X)$, General Topology Appl. 9 (1978), 275-288.

[17] J. T. Rogers, Jr., Applications of a Vietoris-Begle theorem for multi-valued maps to the cohomology of hyperspaces, Michigan Math. J. 22 (1975), 315-319.

[18] A. H. Wallace, Algebraic topology, homology and cohomology, W. A. Benjamin, New York, 1970.

[19] L. E. Ward, Jr., Extending Whitney maps, Pacific J. Math. 93 (1981), 465-469.

[20] G. T. Whyburn, Analytic Topology, AMS, New York, 1942.

S. Macías

Instituto de Matemáticas

Universidad Nacional Autónoma de México

Circuito Exterior, Ciudad Universitaria

México D. F., C. P. 04510

México

E-mail: sergiom@matem.unam.mx

C. Piceno

Instituto de Matemáticas

Universidad Nacional Autónoma de México

Circuito Exterior, Ciudad Universitaria

México D. F., C. P. 04510

México

E-mail: cesarpicman@hotmail.com

Received: 11.1.2012.

Revised: 15.5 .2012 . \& 27.8.2012. 